\title{
SIMULTANEOUS 2D SINGLE-SHOT IMAGING OF OH CONCENTRATIONS AND TEMPERATURE FIELDS IN AN SI ENGINE SIMULATOR
}

\author{
A. ORTH, V. SICK, AND J. WOLFRUM \\ Physikalisch-Chemisches Institut, Ruprecht-Karls-Universität Heidelberg \\ Im Neuenheimer Feld 253, 69120 Heidelberg Germany \\ AND \\ R. R. MALY AND M. ZAHN \\ Daimler-Benz AG, Aero- and Thermodynamics \\ Stuttgart, Germany
}

\begin{abstract}
Two-dimensional (2D) temperature measurements in engines are required for the detailed understanding of the combustion process. Because of the unsteady and turbulent nature of engine combustion, these measurements must be performed on a single-shot basis to obtain cycle-resolved results. Details of the flame front structure are also important to know for the numerical modeling of internal combustion processes. Simultaneous measurements of temperature and hydroxyl radical distributions provide such data sets. The combustion of methane in a fully transparent square piston engine was studied with a combination of $2 \mathrm{D}$ laser-induced fluorescence of hydroxyl radicals and $2 \mathrm{D}$ Rayleigh scattering. A tunable $\mathrm{KrF}$ excimer laser at $248 \mathrm{~nm}$ was used for the measurements. The wavelength of the laser was tuned to excite the $\mathrm{P}_{2}(8)$ transition in the $\mathrm{OH}(3,0)$ band of the A-X system. Spectral filtering of the resulting fluorescence to detect solely the fluorescence from the $(3,2)$ band significantly reduces the effect of collisional quenching imposed to the fluorescence of the predissociating $v^{\prime}=3$ level by vibrational energy transfer (VET) to lower vibrational levels. Using a second camera, which only records the Rayleigh signals after appropriate filtering, allows the simultaneous measurement of temperature fields with a single laser. The analysis of corresponding $\mathrm{OH}$ and temperature images allowed the decision that the combustion conditions are in a regime where the flamelet approach for modeling is appropriate. Good agreement with model predictions has been achieved for the peak temperatures.
\end{abstract}

\section{Introduction}

While multidimensional laser sheet techniques gained increased popularity during the last years $[1,2]$, there is still a challenge for further development of these techniques. One task will be to perform more quantitative measurements, while another one will be to apply the techniques to environments not yet studied. Additionally, there will be increased interest in combining different techniques in the sense of quantitative measurements and in correlating different quantities, like temperature and nitric oxide or flow and concentrations, etc. Especially, nonintrusive measurements in engines are important for an improved understanding of fuel/air mixing [3-6]. Whereas combined multidimensional measurements of different quantities in flames have been performed in a number of objects $[7,8]$, the applications for engines are not well established. Measuring two different quantities usually requires two different wavelengths and, most of the time, two different lasers, e.g., for the simultaneous measurement of fuel and $\mathrm{OH}$ concentration distributions [6]. However, if the excitation wavelength is the same for different molecules or different processes, spectral filtering of the signals can achieve two or more results from a single laser pulse. Using exciplex formation and laser-induced fluorescence, liquid and vapor phases can be distinguished [9]. This is also possible with a combination of Mie scattering and laser-induced fluorescence [5]. Combining Rayleigh scattering and laser-induced fluorescence yields temperature and species concentrations [8]. This technique of simultaneous measurements of Rayleigh scattering and laser-induced fluorescence of hydroxyl radicals has now been applied to measurements in an engine simulator. Rayleigh measurements in engines were already used to measure mixture fractions [10], but no measurements during the combustion phase have been performed there. The combination of temperature and hydroxyl radical concentration measurements is very useful if details of the flame fronts have to be analyzed. For example, deviations from the assumptions of the flamelet model can be measured with this technique [8]. 


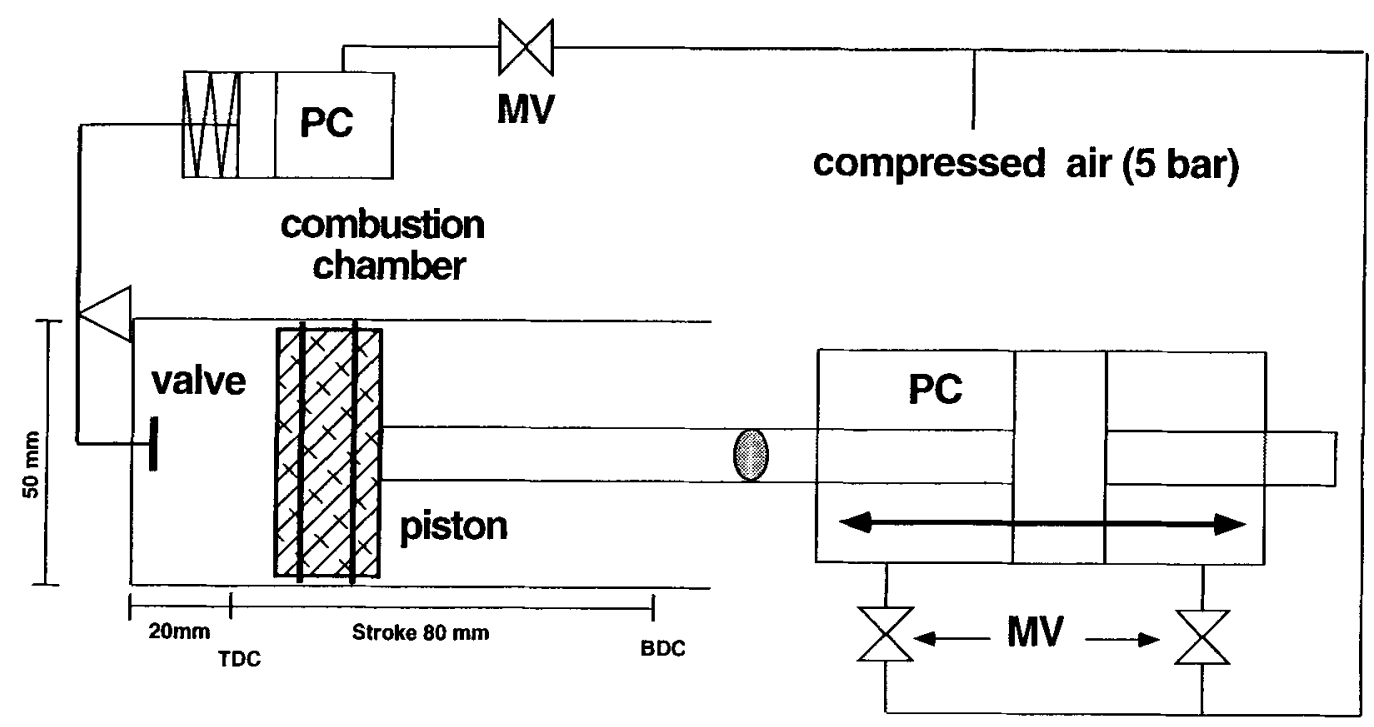

FIG. I. Schematic setup of the pneumatic SI engine simulator. An electronic circuit controls the timing of the piston movement (PC: pneumatic control; MV: magnetic valve).

\section{Experimental}

We investigated the combustion process in a fully transparent SI engine simulator [11]. The cylinder of this engine is built entirely out of quartz plates. The square piston has a cross section of $50 \times 50 \mathrm{~mm}$. The piston movement was controlled by a pneumatic pressure system, which was used to simulate engine speeds of approximately $500 \mathrm{rpm}$. The compression ratio was adjusted to $1: 5$ with a stroke of $80 \mathrm{~mm}$. Cycle-resolved pressure traces were measured with a pressure transducer (Kistler). Methane/air mixtures were stored in a cylinder and were injected through a single valve that was also used as an exhaust valve. As Fig. 1 shows, the engine was operated in a horizontal arrangement.

It is desirable to use short UV wavelengths for Rayleigh scattering since the Rayleigh signal increases with $1 / \lambda^{4}$. For this reason, we used a tunable $\mathrm{KrF}$ excimer laser. The high pulse energy of this laser also allows the two-dimensional (2D) imaging of hydroxyl radicals. Because of the predissociation of the $v^{\prime}=3$ level, quenching has less influence on the signals, and the small Frank-Condon factor of the emission bands is partially compensated at elevated pressures as a result of less quenching losses but higher densities. If the $(3,0) \mathrm{P}_{2}(8)$ transition of $\mathrm{OH}$ is excited at $248.456 \mathrm{~nm}$, no temperature influence has to be taken into account to correct for changes in the population density given by the Boltzmann distribution. In the range 1300-3000 K, the fraction of molecules in $N^{\prime \prime}=8$ is constant within $10 \%$. As has been pointed out several times [12-14], it is crucial to filter the fluorescence signals properly in or- der to avoid detection of fluorescence from $v^{\prime}<3$. These levels are populated via vibrational energy transfer (VET) and suffer from quenching. Thus, the measured signals would be sensitive to quenching. We used a bandpass filter with a center wavelength of $297 \pm 6 \mathrm{~nm}$. This filter transmits the fluorescence of the $(3,2)$ vibrational band. Also important to obtain high signal levels is a camera lens with a large aperture. The achromatic lens systems we used had an $\mathrm{f \#}=2$, thus increasing the signal strengths remarkably compared to the commonly used systems. Since we were interested in measuring the $\mathrm{OH}$ distribution and the temperature fields simultaneously, we used a second camera to record Rayleigh scattering. Figure 2 shows the experimental setup where the two cameras were mounted on the opposite sides of the engine. The Rayleigh signals had to be filtered from any fluorescence light. We found it appropriate to use a mirror coated for $248 \mathrm{~nm}$ and a color glass filter (Schott UG 5). Careful alignment of the two camera/lens/filter systems is necessary to make sure that the same plane is monitored by both cameras. As shown in Fig. 2, the laser illuminates a horizontal plane in the engine. The beam was rotated with a Dove prism and collimated to a light sheet of less than $300 \mu \mathrm{m}$ in thickness. It is crucial to make sure that the sheet is not thicker because, otherwise, important details of the flame front, like superequilibrium concentrations of hydroxyl radicals [11], cannot be measured. The Bunsen burner shown in Fig. 2 was used to control the proper tuming of the laser to the $P_{2}(8)$ transition by maximizing the OH-LIF signal from this flame using camera 3 . Special care 


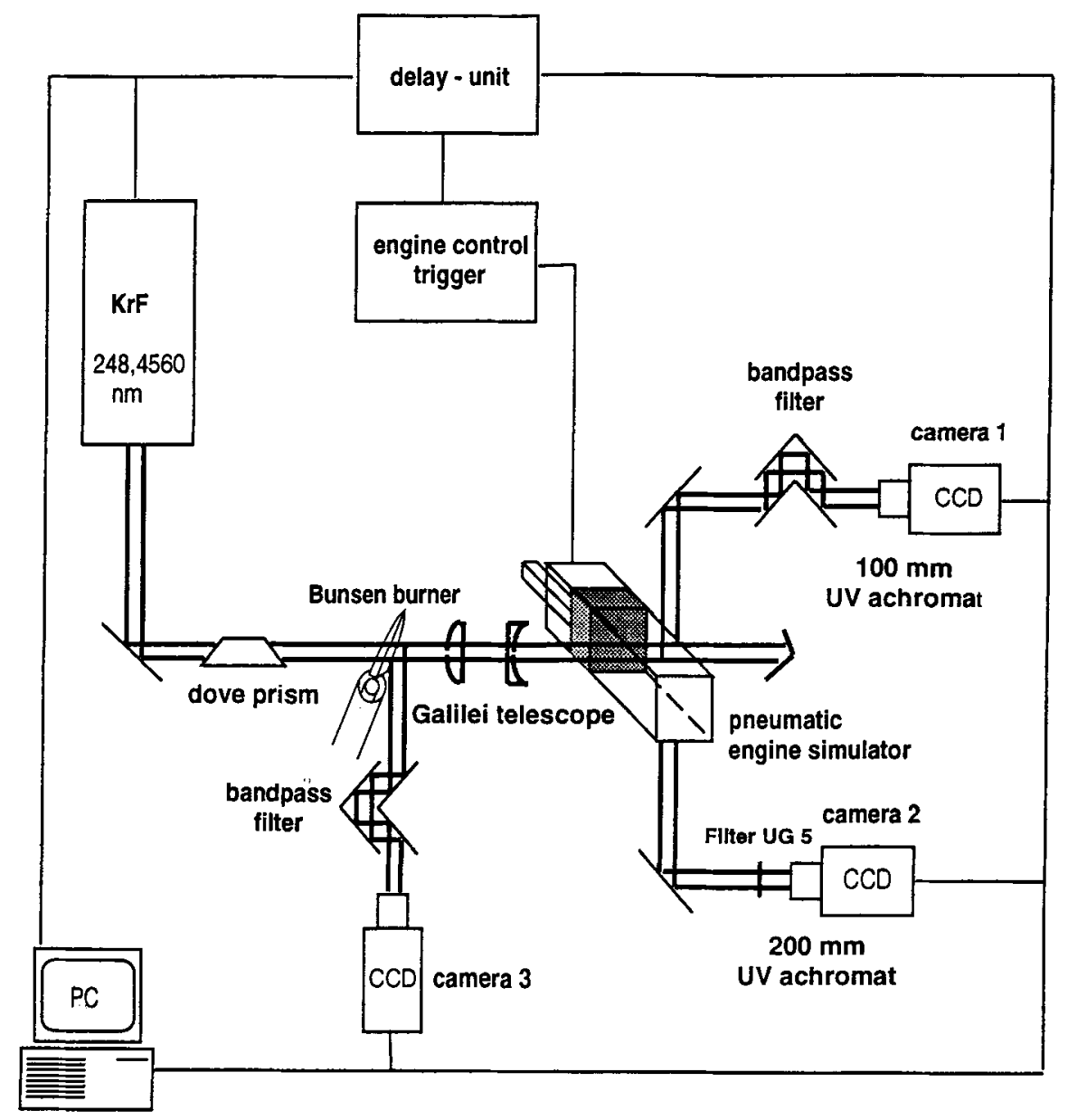

FIG. 2. Experimental setup for simultaneous Rayleigh and LIF measurements in an SI engine simulator. A tunable $\mathrm{KrF}$ excimer laser, tuned to the $(3,0) \mathrm{P}_{2}(8)$ transition of hydroxyl radicals, was used for the measurements.

had to be taken to avoid scattering of the laser beam at any surface of the experiment. A certain distance from the walls had to be maintained for the Rayleigh measurements, whereas it was possible to follow the $\mathrm{OH}$ concentration up to the walls of the piston and the cylinder.

All signals were recorded with image-intensified charge-coupled device (CCD) cameras (Proxitronic Nanocam II) with gate times of 50 ns. Using frame grabber boards (Matrox MVP AT), the signals were digitized and processed, as will be described later in this paper. It is important to mention that cameras of the type we used have a fixed time for light detection given by the frame-transfer process. This frame transfer is set by the video frequency and cannot be altered. If signals are recorded at inappropriate times, the measured intensity is lower. For this reason, it is necessary to make sure either that images that are measured near frame transfers are rejected or that the experiment's timing is coupled to the frame-transfer frequency. Of course, this is not possible for many experiments in engines. But for the engine we used, we could set an external trigger for the engine operation and then couple cameras, lasers, and the engine to the frame-transfer process.

Whereas the interpretation of the laser-induced Aluorescence (LIF) data is more straightforward to obtain information about thickness, curvature, and movement of the flame front, it is more complicated to analyze the Rayleigh images. The only corrections that have been made for the $\mathrm{OH}$ images are for irregular laser beam profiles and absolute energy fluctuations. For the Rayleigh images, a series of corrections and calibration procedures has to be used before temperature fields are obtained. Rayleigh scattering is proportional to the total particle number $N$. Thus, by using the ideal gas assumption, it is proportional to the pressure $p$ and the cross section $\sigma_{s}$, 
and is inversely proportional to the temperature [Eq. (1)]. It is also proportional to the laser energy $I_{0}$ and the probe volume $V$ :

$$
R_{\mathrm{Ray}}=V I_{0} p N \sigma_{s} / R T .
$$

However, the cross section for Rayleigh scattering is different for different molecules, and the total cross section is the sum of the cross section for each type of molecule in a mixture weighted with its mole fraction [15]. This means that if the gas composition changes, the cross section changes and makes it impossible to obtain accurate temperature data without knowing the gas composition. Fortunately, for the methane/air mixtures investigated here, the variation in the total cross section between burnt and unburnt gases is smaller than $2 \%$ [8].

With this, the temperature at a point with coordinates $(x, y)$ can be calculated from the measured Rayleigh intensity $R(x, y)$

$$
\begin{aligned}
T(x, y) & =T_{0} c R_{c}(x, y) / R(x, y) \\
c & =\sigma_{\text {flame }} / \sigma_{\text {fuel }} .
\end{aligned}
$$

Here, $T_{0}$ is a reference temperature, where a calibration signal $R_{c}(x, y)$ is measured. If this signal is measured in ambient air, the calibration constant $c$ takes into account that the cross section for air is different than that from the methane/air mixture. However, we chose another approach. We measured the reference signal with the methane/air in the engine at the crank angle where the measurements were taken, but without ignition. The temperature there can be calibrated against air/ambient pressure conditions, or it can be calculated adiabatically from thermodynamical data. This procedure has the advantage that the dynamic range of the signals and the camera match much better. Since heating increases the pressure during fired cycles, at corresponding crank angles, the pressure ratio has to be taken into account during data analysis. Equation (2) transforms to

$$
T(x, y)=T_{0} c R_{c}(x, y) / R(x, y)\left(P / P_{0}\right)\left(I / I_{0}\right) .
$$

Additional processing of the data is necessary to obtain temperatures. Any residual light that is scattered from surfaces distorts the measurements. To take this into account, images were taken where the engine was flooded with helium. The Rayleigh cross section of helium is almost negligible, and therefore, the remaining measured intensity is scattered light. These images have to be subtracted from the measured Rayleigh images. Also, an image taken with the camera lens covered was taken and subtracted from all Rayleigh images. This background correction is necessary to exclude offsets of the digitizers and camera noise. Of course, all images with the exception of the latter one have to be normalized to the laser intensity profile. This profile is best determined by taking the reference image for normalization of all other images.

\section{Results}

Temperature and hydroxyl radical distributions have been measured simultaneously with a single laser pulse in a fully transparent SI engine simulator for different stoichiometries. All images shown here are taken in a plane parallel to the piston movement in the middle of the combustion chamber. The mixtures were ignited with a spark plug always located on the right side of the images. Since the cross sections imaged by the two cameras have different sizes, the $\mathrm{OH}$ images were rescaled to match the size of the temperature images. This was done by carefully comparing the image of a scale that was placed in the plane of the laser sheet. It is then possible to compare $\mathrm{OH}$ concentration fields with corresponding temperature fields. The image pairs of Fig. 3 nicely demonstrate how temperature and hydroxyl radical concentration are coupled. Even details of the temperature field also can be recognized in the $\mathrm{OH}$ images. We did not attempt to calibrate our LIF images to absolute values, but this could be done by comparing the measured signals from the engine simulator with signals obtained in a calibrated burner [16]. As was pointed out earlier [11], the laser sheet must be thinner than the flame front thickness to be able to resolve the flame front structure properly. Figure 4a shows one of the examples from Fig. 3 in an enlarged view, together with profiles along the white lines indicated in Fig. 4a. Entering the flame front from the unburnt gases, it is obvious that temperature is increasing before the $\mathrm{OH}$ concentration rises. From profiles like this, a (90-10\%) flame front thickness was determined to be $300 \pm 100 \mu \mathrm{m}$. This value represents the smallest flame front thicknesses found in the images, indicating that here the flame front was illuminated perpendicularly by the light sheet. The flamelet assumption is only valid if temperature and hydroxyl radical concentrations are correlated, as in laminar flames. The strong correlation found in our engine experiments confirms the validity of the flamelet approach for the conditions studied here. As expected, the spatial gap between temperature rise and $\mathrm{OH}$ concentration rise becomes less at higher pressures. Here, $300 \mu \mathrm{m}$ were measured at $P=5.3 \mathrm{bar}$, compared to $500-700 \mu \mathrm{m}$ at atmospheric pressure flames [8]. Although we did not attempt to measure absolute $\mathrm{OH}$ concentrations, the relative concentration profiles indicate superequilibrium values of hydroxyl radicals in the flame front, in agreement with previous measurements and modeling results [17]. This result is based on the fact that, in the exhaust gases, $\mathrm{OH}$ reaches equilibrium 

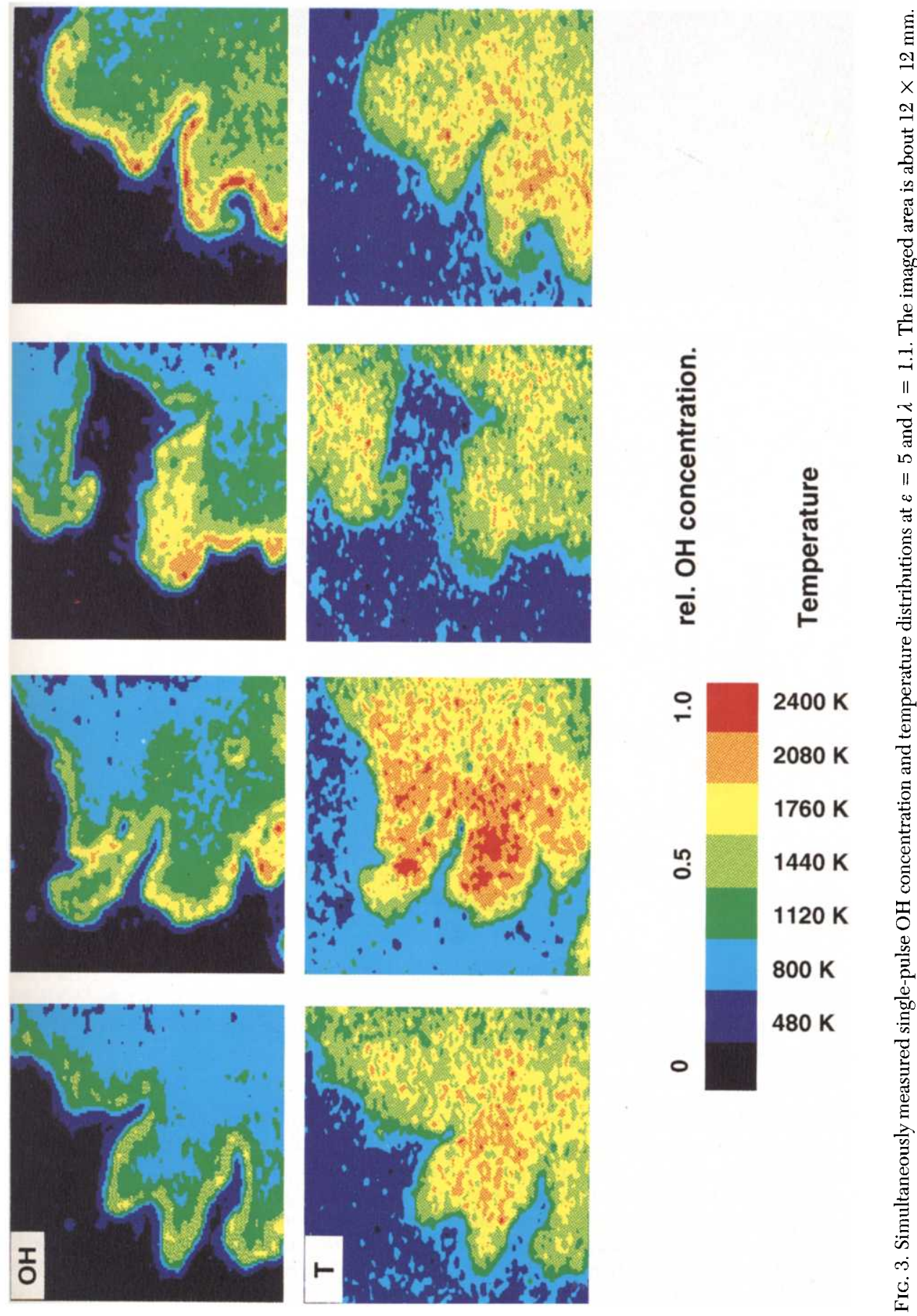


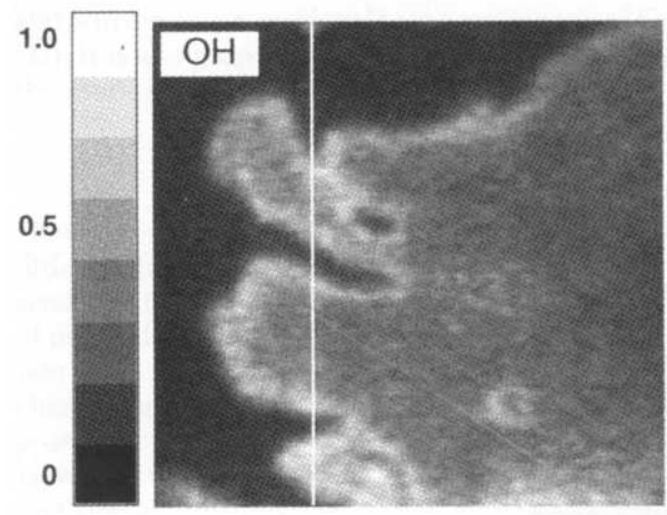

Relative $\mathrm{OH}$ Concentration
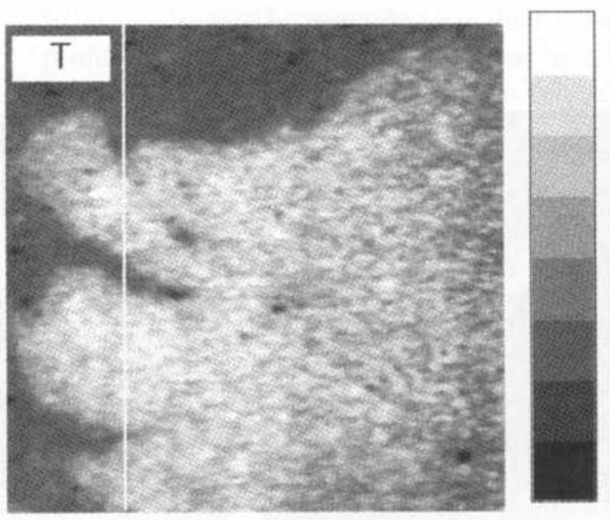

1950

1770

1590

1400

1220

860

680

500

\section{Temperature}

(a)

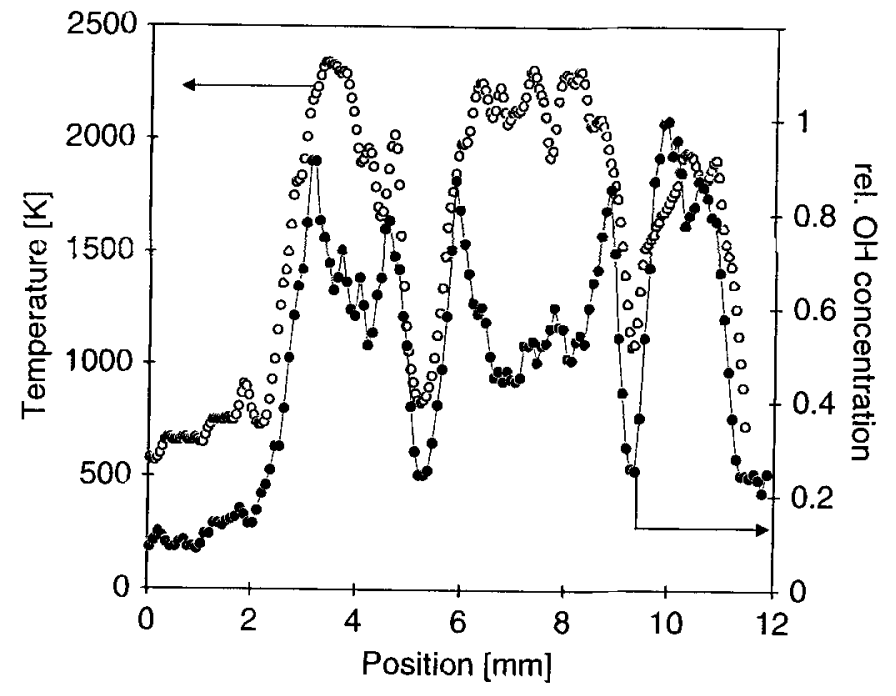

(b)

FIG. 4. (a) Enlarged example from Fig. 3. The positions for the vertical profiles of Fig. $4 \mathrm{~b}$ are indicated as white bars. (b) Profiles of $\mathrm{OH}$ concentration and temperature at $\varepsilon=5$ and $\lambda=1.1$. There is a clear separation between temperature rise and increasing $\mathrm{OH}$ concentration on both sides of the profiles, where the image also shows unburnt areas in contrast to the two temperature drops in the middle of the profile.

values, so that the high concentrations in the flame front can be attributed to superequilibrium concentrations. The peak temperatures measured are in agreement with the predicted peak temperatures of $2300 \mathrm{~K}$. If comparisons with modeling results are considered, not only single-pulse measurements are required for details of the combustion process, but aiso average temperature and concentration distributions are necessary. The presently used multidimensional codes for modeling combustion processes in engines always predict averaged data. To account for this demand, we calculated averaged temperature and hydroxyl radical concentrations. Figure 5a shows the averaged $\mathrm{OH}$ and temperature distribution for a lean mixture with $\lambda=1.1$ and a compression ratio of 1:5. The engine simulator was operated at conditions corresponding to $500 \mathrm{rpm}$. The averaged peak temperature data were determined to be $1790 \mathrm{~K}$ with a statistical variance of $\pm 150 \mathrm{~K}$. The estimated accuracy is $15 \%$ for our measurements. For a mixture with $\lambda=1.2$, the averaged peak temperature is higher. Temperatures of $1990 \mathrm{~K} \pm 120 \mathrm{~K}$ were measured for these conditions. The average was calculated from 15 single-shot images, with averaging 


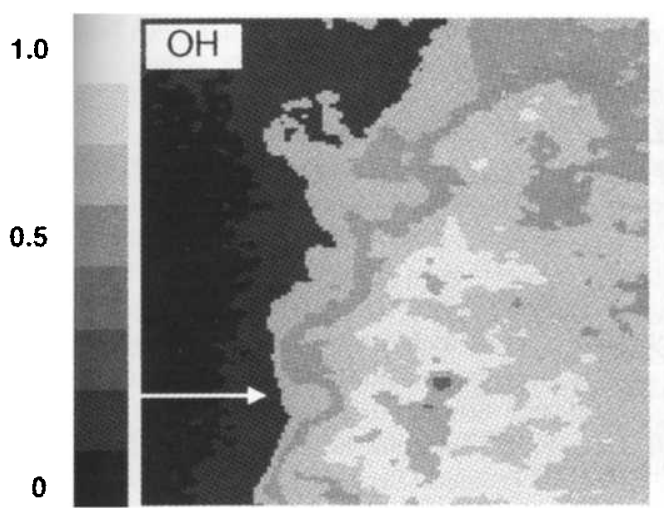

Relative $\mathrm{OH}$ Concentration
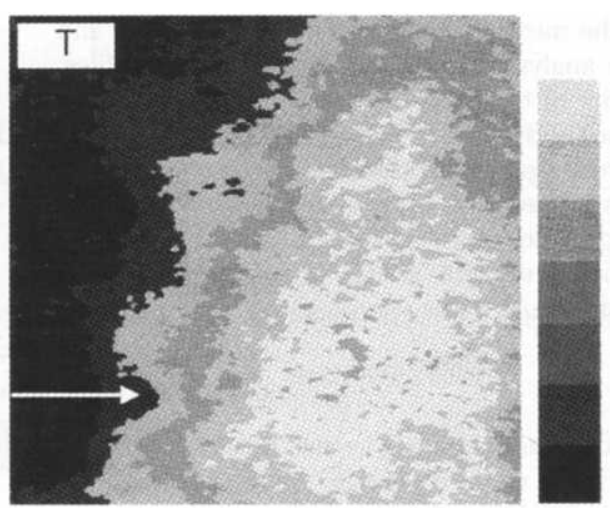

$1950 \mathrm{~K}$

$1770 \mathrm{~K}$

$1590 \mathrm{~K}$

$1400 \mathrm{~K}$

$1220 \mathrm{~K}$

$860 \mathrm{~K}$

$680 \mathrm{~K}$

$500 \mathrm{~K}$

\section{Temperature}

(a)

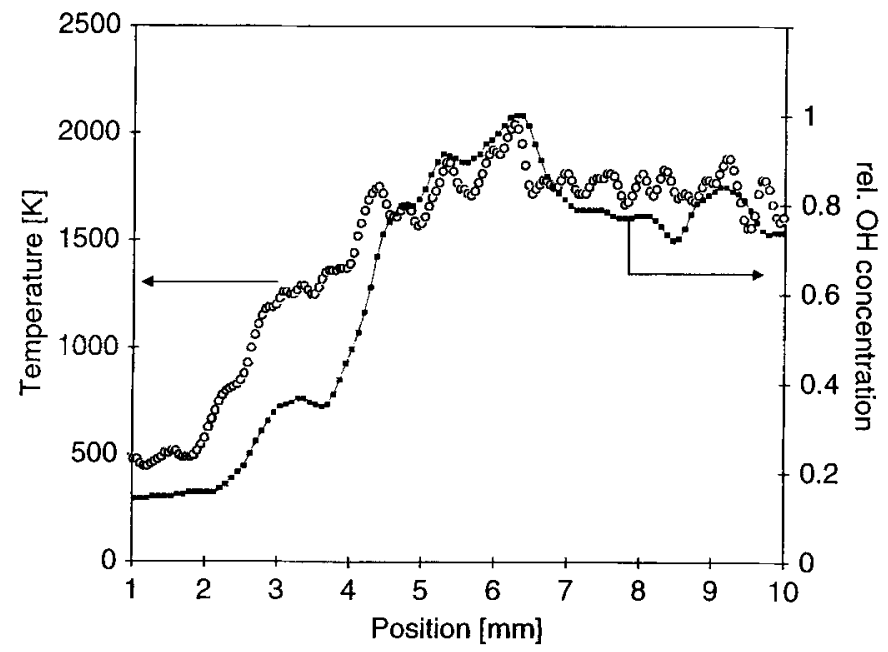

(b)

FrG. 5. (a) Averaged hydroxyl radical concentration and temperature distribution for $\varepsilon=5$ and $\lambda=1$.1. The image is the average of 25 single-pulse measurements. (b) Profiles from the averaged distributions of Fig. $5 \mathrm{a}$.

over a $56 \times 56$ matrix in areas of the highest temperatures. The positions of the profiles shown in Fig. $5 \mathrm{~b}$ are indicated in Fig. 5a. Major features of the flame front are given in both profiles. However, the spatial gap of the temperature and hydroxyl radical concentration rise in the flame front is larger than it is in the single-pulse measurements. If the coupling of both quantities has to be determined to decide about the validity of the flamelet concept for a given combustion process, single-pulse measurements are required. In the sense of obtaining ensemble average data for comparison with model predictions, it would be useful to acquire the data at fixed intervals for the, e.g., peak pressure. This procedure would reduce the influence of cyclic variability on the results.

\section{Conclusions}

We demonstrated that it is possible to use 2D Rayleigh scattering for temperature measurements in an SI engine simulator. Moreover, within a single laser pulse, the temperature and hydroxyl radical concentration distribution were measured using a tunable $\mathrm{KrF}$ excimer laser and two image-intensified CCD cameras. A significant dependence of the tempera- 
ture on the mixture composition was measured, and from the analysis of $\mathrm{OH}$ and temperature profiles, the validity of the flamelet assumption for the conditions under study has been confirmed. Modeling results from previous studies [17] are confirmed by our measurements. Averaged data have been calculated to provide a data set for comparison with multidimensional models, which usually predict averaged data [18].

\section{Acknowledgments}

This work was sponsored by the Bundesministerium für Forschung und Technologie under Contract No. TECFLAM 03E6325L and Daimler-Benz AG, Stuttgart.

\section{REFERENCES}

1. Hanson, R. K., and Seitzman, J. M., in Instrumentation for Flows with Combustion (A. M. P. K. Taylor, Ed.), Academic Press, London, 1993.

2. Arnold, A., Bräumer, A., Buschmann, A., Decker, M., Dinkelacker, F., Heitzmann, T., Orth, A., Schäfer, M., Sick, V., and Wolfrum, J., Ber. Bunsenges. Phys. Chem. 97:1650-1661, 1993.

3. Baritaud, T. A., and Green, R. M., SAE Technical Paper Series 860025, Philadelphia, 1986.

4. Felton, P. G., Mantzaras, J,, Bardsley, E. A., and Bracco, F. V., SAE Technical Paper Series 872074 , Philadelphia, 1987.

5. Arnold, A., Dinkelacker, F., Heitzmann, T., Monkhouse, P., Schäfer, M., Sick, V., Wolfrum, J., Hentschel, W., and Schindler, K.-P., Twenty-Fourth Symposium (International) on Combustion, The Combustion Institute, Pittsburgh, 1992, pp. 16051612.

6. Arnold, A., Buschmann, A., Cousyn, B., Decker, M.,
Sick, V., Vannobel, F., and Wolfrum, J., SAE Technical Paper Series 9326966, Philadelphia, 1993.

7. Long, M. B., Levin, P. S., and Fourgette, D. C., Opt. Lett. 10:267-269 1985.

8. Dinkelacker, F., Buschmann, A., Schäfer, M., and Wolfrum, J., Joint Meeting of the British and German Sections of the Combustion Institute, British Section of the Combustion Institute, Cambridge, UK, 1993, pp. 295-298.

9. Melton, L. A., and Verdieck, J. F., Twentieth Symposium (International) on Combustion, The Combustion Institute, Pittsburgh, 1984, pp. 1283-1290.

10. Zhao, F. -Q., Taketomi, M., Nishida, K., and Hiroyasu, H., SAE Technical Paper Series 932641, Philadelphia, 1993.

11. Suntz, R., Becker, H., Monkhouse, P., and Wolfrum, J., Appl. Phys. B 47:287-293 1988.

12. Andresen, P., Bath, A., Gröger, W., Lülf, H. W., Meijer, G., and terMeulen, J. J., Appl. Opt. 27:365-378 1988.

13. Arnold, A., Lange, B., Bouché, T., Heitzmann, T., Schiff, G., Ketterle, W., Monkhouse, P., and Wolfrum, J., Ber. Bunsenges. Phys. Chem. 96:1388-1393 1992.

14. Steffens, K. L., Jeffries, J. B., and Crosley, D. R., Opt. Lett. 18:1355-1357 1993.

15. Dibble, R. W., and Hollenbach, R. E., Eighteenth Symposium (International) on Combustion, The Combustion Institute, Pittsburgh, 1981, pp. 1489-1499.

16. Sick, V., Arnold, A., Dießel, E., Dreier, T., Ketterle, W., Lange, B., Wolfrum, J., Thiele, K. -U., Behrendt, F., and Warnatz, J., Twenty-Third Symposium (International) on Combustion, The Combustion Institute, Pittsburgh, 1990, pp. 495-501.

17. Becker, H., Arnold, A., Suntz, R., Monkhouse, P., Maly, R. R., and Pfister, W., Appl. Phys. B 50:473-478 1990.

18. Herweg, R., and Maly, R. R., SAE Technical Paper Series 922243, Philadelphia, 1992. 